\title{
Impact of Adjuvant Chemotherapy Completion on Oncologic Outcomes in ypTNMstage 2 Rectal Cancer Patients
}

\author{
Youn Young Park ${ }^{1}$, Kang Young Lee ${ }^{2,3}$, Nam Kyu Kim², Sat Byol Lee ${ }^{2,3}$, Ga Ram Kim ${ }^{4}$ Byung Soh Min ${ }^{2,3}$, \\ Seong-Taek $\mathrm{Oh}^{1}$ \\ ${ }^{1}$ Department of Surgery, Uijeongbu St. Mary's Hospital, College of Medicine, The Catholic University of Korea, Seoul; ${ }^{2}$ Department of Surgery, \\ Yonsei University College of Medicine, Seoul; ${ }^{3}$ OpenNBI Convergence Technology Laboratory, Avison Biomedical Research Centre, Yonsei \\ University College of Medicine, Seoul; ${ }^{4}$ Department of Radiology, Inha University Hospital, Inha University School of Medicine, Incheon, Korea
}

Purpose: Adjuvant chemotherapy $(\mathrm{aCT})$ in rectal cancer patients who have undergone curative resection after neoadjuvant chemoradiation (nCRT) is controversial. We aimed to investigate the benefits of using aCT and the clinical impact of completing aCT in ypstage 2 rectal cancer patients.

Methods: We retrospectively reviewed clinicopathological data from patients who had undergone radical resection after nCRT between January 2006 and December 2012. In total, 152 patients with ypT3/4N0M0 rectal cancer were included. Of these patients, 139 initiated aCT, while 13 did not receive aCT (no-aCT). Among those who received aCT, 132 patients completed their planned cycles (aCT-completion) whereas 7 did not (aCT-incompletion). All patients received longcourse chemoradiation; a 5-fluorouracil-based regimen was used for nCRT in most patients. The prognostic factors affecting disease-free survival (DFS) and overall survival (OS) were analyzed.

Results: The median follow-up duration was 41 months. Demographic data did not differ significantly among the 3 groups. In multivariate analysis, open surgery, a tumor size $>2 \mathrm{~cm}$, retrieval of $<12$ lymph nodes, circumferential resection margin (CRM) positivity and aCT incompletion were independent prognostic factors for poor DFS. Old age ( $\geq 60$ years), open surgery, CRM positivity, aCT incompletion, and lack of aCT initiation compared to aCT completion were independent prognostic factors for poor OS.

Conclusion: In ypstage 2 rectal cancer patients, aCT after nCRT and total mesorectal excision affected both DFS and OS; however, only patients who completed planned aCT exhibited survival benefits. Therefore, improving patients' compliance with the completion of aCT is desirable.

Keywords: Rectal neoplasm; Neoadjuvant therapy; Chemoradiotherapy; Adjuvant chemotherap

Received: February 1,2019 • Accepted: March 10, 2019

Correspondence to: Byung Soh Min, M.D.

Department of Surgery, Yonsei University College of Medicine,

50-1 Yonsei-ro, Seodaemun-gu, Seoul 03722, Korea

Tel: +82-2-2228-2100, Fax: +82-2-313-8289, E-mail: bsmin@yuhs.ac

ORCID code: https://orcid.org/0000-0003-0180-8565

Co-correspondence to: Seong-Taek Oh, M.D.

Department of Surgery, Uijeongbu St. Mary's Hospital, College of Medicine, The Catholic University of Korea, 271 Cheonbo-ro, Uijeongbu 11765, Korea Tel: +82-31-820-3998, Fax: +82-31-847-2717, E-mail: stoh@catholic.ac.kr ORCID code: https://orcid.org/0000-0002-5962-581X

(C) 2019 The Korean Society of Coloproctology

This is an open-access article distributed under the terms of the Creative Commons Attribution NonCommercial License (http://creativecommons.org/licenses/by-nc/4.0) which permits unrestricted noncommercial use, distribution, and reproduction in any medium, provided the original work is properly cited.

\section{INTRODUCTION}

Neoadjuvant chemoradiation (nCRT) followed by total mesorectal excision (TME) has been established as a standard therapeutic strategy for locally advanced rectal cancer, based on evidence from randomized controlled trials (RCTs) [1-6]. However, adjuvant chemotherapy $(\mathrm{aCT})$ following TME after $\mathrm{nCRT}$ is an extrapolation of this therapeutic strategy for colon cancer $[7,8]$. The national as well as National Comprehensive Cancer Network guidelines recommend $\mathrm{aCT}$ after $\mathrm{nCRT}$ and $\mathrm{TME}$, irrespective of the final pathological report [9].

Recent studies have had contradictory results on the benefits of 
aCT after nCRT and TME, but there has been increasing evidence that good responders displaying node negativity after nCRT do not benefit from aCT [10-17]. Therefore, some investigators have suggested that aCT should not be routinely applied after nCRT in good responders [10-12]. However, even if node negativity is confirmed after nCRT and TME, when standard treatment strategies for high-risk stage 2 colon cancer patients are considered, concerns may arise that omitting aCT will lead to unfavorable oncologic outcomes in patients with advanced T stages (ypT3/4).

Importantly, studies suggesting a lack of benefit from aCT after nCRT in good responders only included patients who had completed their scheduled aCT, or did not clarify whether the patients had completed their scheduled aCT [10-13]. Therefore, these patients were not representative of real-world patients, considering the poor compliance with aCT after nCRT and TME in rectal cancer patients; thus, these studies may have underestimated the effects of aCT. Since previous studies have demonstrated that aCT incompletion worsens the prognosis of colon cancer patients, there may be important clinical implications to investigating the oncologic impact of aCT incompletion after nCRT and TME, along with aCT initiation.

Therefore, this study investigated the benefits of aCT initiation and the oncologic impact of aCT completion after nCRT and TME in ypstage 2 (ypT3/4N0M0) rectal cancer patients.

\section{METHODS}

From a prospectively collected database, we identified patients who had undergone curative resection after nCRT from January 2006 to December 2012 and who exhibited ypN0 on their final pathological reports. Patients who had histories of malignancies in other organs within 5 years, had been diagnosed with hereditary colorectal cancer or recurrent rectal cancer, had undergone palliative surgery or had been treated postoperatively with additional radiotherapy (RT) were excluded. Patients who underwent surgery or switched treatment regimens for palliative purposes due to early local recurrence (LR) or distant metastasis (DM) during the 4 months of planned aCT were also excluded from the final analysis. This study was approved by the Institutional Review Board of Severance Hospital (4-2018-1130) for exemption of informed consent.

In total, 152 patients were included, of whom 139 received aCT and 13 did not. We retrospectively reviewed the patients' electronic medical data to obtain each patient's age, sex, body mass index, American Society of Anesthesiologists physical status classification, preoperative carcinoembryonic antigen (CEA) level, tumor level, clinical stage, nCRT regimen, name and type (minimally invasive [laparoscopic or robotic] $v s$. open) of surgery, anastomotic leakage, hospital stay, histology, tumor regression grade (Mandard grade), pathological stage, tumor size, number of retrieved nodes, circumferential resection margin (CRM), lymphovascular invasion, radicality of resection, initiation and comple- tion of aCT, and aCT regimen. Variables were compared among patients who did not receive aCT (no-aCT), patients who initiated but did not complete aCT (aCT-incompletion) and patients who completed aCT (aCT-completion). We analyzed factors affecting disease-free survival (DFS) and overall survival (OS). Completion of aCT was defined as the completion of scheduled aCT, and incompletion was defined as discontinuation within 4 months of aCT initiation for any reason.

For pretreatment workups, a digital rectal exam, colonoscopy, abdominopelvic computed tomography (CT), pelvic magnetic resonance imaging (MRI) and chest CT were conducted, and the clinical stage was determined based on the results of these pretreatment workups. Patients with clinical T3/4Nany or TanyN+ cancers were treated with nCRT. For the nCRT protocol, the majority of patients received 5-fluorouracil (5-FU)-based longcourse RT (5-FU $400 \mathrm{mg} / \mathrm{m}^{2} /$ day and leucovorin [LV] $20 \mathrm{mg} / \mathrm{m}^{2} /$ day for 5 days on days $1-5$ and days 29-33 or capecitabine 825 $\mathrm{mg} / \mathrm{m}^{2}$ twice daily for 5 days/wk during long-course RT [45-50.4 Gy in 25-28 fractions]). An irinotecan plus S-1 regimen was also used in some patients.

TME was performed within 4-8 weeks of the completion of nCRT. Within 4-6 weeks of surgery, aCT was administered. The regimens for aCT included a bolus injection of FL (5-FU $400 \mathrm{mg} /$ $\mathrm{m}^{2} /$ day and LV $20 \mathrm{mg} / \mathrm{m}^{2} /$ day for 5 days every 4 weeks for $4 \mathrm{cy}-$ cles) or oral capecitabine $\left(1,250 \mathrm{mg} / \mathrm{m}^{2}\right.$ twice daily for 2 weeks every 3 weeks for 6 cycles) and a FOLFOX regimen (infusion of oxaliplatin $85 \mathrm{mg} / \mathrm{m}^{2}$ and LV $200 \mathrm{mg} / \mathrm{m}^{2}$ followed by a bolus of 5 -FU $400 \mathrm{mg} / \mathrm{m}^{2}$ and continuous infusion of 5-FU $2400 \mathrm{mg} / \mathrm{m}^{2}$ for 2 days every 2 weeks for 8 cycles). We defined incompletion of $\mathrm{aCT}$ as administration of aCT for less than 4 months.

The patients were followed up every 3 months for 2 years, and then every 6 months for the next 3 years. Physical examinations and chest $\mathrm{X}$-rays were performed and CEA levels were assessed at every visit. Abdominopelvic $\mathrm{CT}$ and chest $\mathrm{CT}$ were performed at every 6-month and 1-year follow-up, respectively. Colonoscopies were performed 1,3 , and 5 years after surgery. Liver MRI, pelvic MRI or 18-fluorodeoxyglucose-positron emission tomography was performed as needed. DFS was defined as the time to disease progression; OS was defined as the time interval between surgery and death.

Categorical variables were analyzed by the chi-square test or Fisher exact test, and continuous variables were analyzed by Student t-test or the Mann-Whitney U-test. Kaplan-Meier curves were constructed for OS and DFS, and survival outcomes were compared through a log rank test. Prognostic factors for DFS and OS were analyzed with a Cox regression model. P-values $<0.05$ were considered statistically significant. IBM SPSS Statistics ver. 23.0 (IBM Co., Armonk, NY, USA) was used for statistical analyses.

\section{RESULTS}

In total, 152 patients were included in the analysis. The median 
follow-up duration was 41 months (interquartile range [IQR], $30-56$ months). The mean age of the patients was $58.8 \pm 11.53$ years, and 106 patients were male (69.7\%). In total, 116 patients received 5-FU-based nCRT (76.3\%).

In 13 patients, aCT was not initiated after nCRT and TME: 6 patients refused aCT, 4 developed postoperative complications such as a rectovaginal fistula, anastomotic leakage or surgical site infection, 1 developed a brain infarction after surgery, 1 developed hepatic encephalopathy after surgery, and 1 developed severe hand-foot syndrome after nCRT. In the remaining 139 patients, aCT was initiated after nCRT and TME. Among them, 132 patients completed their planned aCT schedules, whereas 7 did not. Of these 7, 2 stopped receiving aCT due to severe side effects of the chemotherapeutic agents, 1 refused to complete the planned schedule and 4 discontinued their aCT for reasons that were not recorded.

The patients' demographics are shown in Table 1. There were no significant differences in patient characteristics among the 3 groups. The 5 -year DFS and OS rates of patients with ypstage 2 cancer were $72.5 \%$ and $85.3 \%$, respectively. The 5 -year DFS rate was $74.8 \%$ in the aCT-completion group, $42.9 \%$ in the aCT-incompletion group and $58.9 \%$ in the no-aCT group; the DFS curves differed significantly with a P-value of 0.029 (Fig. 1A). Regarding the sites of recurrence, the 5-year LR-free survival rates were $91.6 \%, 57.1 \%$, and $64.3 \%(\mathrm{P}=0.001)$, and the 5-year DMfree survival rates were $81.1 \%, 57.1 \%$, and $59.3 \%(\mathrm{P}=0.155)$ in the aCT-completion, aCT-incompletion and no-aCT groups, respectively. The 5-year OS rate was $88.7 \%$ in the aCT-completion group, $53.6 \%$ in the aCT-incompletion group and $76.9 \%$ in the no-aCT group; the OS curves differed significantly with a P-value $<0.001$ (Fig. 1B).

Univariate analysis of the prognostic factors for DFS revealed that low rectal cancer, abdominoperineal resection (APR), open surgery, a tumor size $>2 \mathrm{~cm}$ at final pathology, retrieval of $<12$ lymph nodes, CRM positivity ( $\leq 1 \mathrm{~mm}), \mathrm{R} 1$ resection and aCT incompletion were associated with poor DFS. Multivariate analysis indicated that open surgery (hazard ratio [HR], 2.243; 95\% confidence interval [CI], 1.099-4.578); $\mathrm{P}=0.026$ ), a tumor size $>2 \mathrm{~cm}$ (HR, 4.168; 95\% CI, 1.661-10.461; $\mathrm{P}=0.002$ ), retrieval of $<12$ lymph nodes (HR, 2.438; 95\% CI, 1.215-4.890; P = 0.012), CRM positivity (HR, 3.673; 95\% CI, 1.579-8.542; $\mathrm{P}=0.003)$ and aCT incompletion compared to aCT completion (HR, 7.052; 95\% CI, 2.323-21.410; $\mathrm{P}=0.001$ ) were independent prognostic factors for poor DFS (Table 2).

Univariate analysis of the prognostic factors for OS revealed that old age ( $\geq 60$ years), low rectal cancer, a clinical stage of T3/4, APR, open surgery, a long hospital stay (>10 days), a pathologic stage of ypT4, retrieval of $<12$ lymph nodes, CRM positivity, R1 resection, no-aCT initiation compared to aCT initiation, and aCT incompletion or no-aCT initiation compared to aCT completion were associated with a poor prognosis. In multivariate analysis, old age ( $\geq 60$ years) (HR, 3.840; 95\% CI, 1.204-12.251; $\mathrm{P}=0.025)$, open
Table 1. Patient demographics

\begin{tabular}{|c|c|c|c|c|}
\hline Characteristic & Completion & Incompletion & No-aCT & P-value \\
\hline Age $\geq 60 \mathrm{yr}$ & $63(47.7)$ & $3(42.9)$ & $10(76.9)$ & 0.123 \\
\hline Male sex & 94 (71.2) & $6(85.7)$ & $6(46.2)$ & 0.120 \\
\hline $\begin{array}{l}\text { Body mass index } \\
\left(\geq 25 \mathrm{~kg} / \mathrm{m}^{2}\right)\end{array}$ & $26(19.7)$ & $1(14.3)$ & $3(23.1)$ & 0.896 \\
\hline ASA PS classification $\geq$ III & $4(3.0)$ & $0(0)$ & $1(7.7)$ & 0.511 \\
\hline $\begin{array}{l}\text { Preoperative CEA }>5 \mu \mathrm{g} / \mathrm{L} \\
\text { (NA in 1) }\end{array}$ & 50 (38.2) & $3(42.9)$ & $2(15.4)$ & 0.251 \\
\hline $\begin{array}{l}\text { Low rectal cancer } \\
\qquad(<\mathrm{AV} 6 \mathrm{~cm})\end{array}$ & $55(41.7)$ & $4(57.1)$ & $6(46.2)$ & 0.713 \\
\hline$\geq \mathrm{cT3}$ & $127(96.2)$ & $7(100)$ & 12 (92.3) & 0.578 \\
\hline $\mathrm{cN}+$ & 96 (72.7) & $5(71.4)$ & $9(69.2)$ & 0.920 \\
\hline nCRT regimen & & & & 0.116 \\
\hline 5-FU-based & $99(75.0)$ & $4(57.1)$ & $13(100)$ & \\
\hline TS-1 \& irinotecan & $31(23.5)$ & $3(42.9)$ & $0(0)$ & \\
\hline Unknown & $2(1.5)$ & $0(0)$ & $0(0)$ & \\
\hline Operation & & & & 0.758 \\
\hline Sphincter-saving & $112(84.8)$ & $7(100)$ & $11(84.6)$ & \\
\hline APR & 20 (15.2) & $0(0)$ & $2(15.4)$ & \\
\hline Minimally invasive surgery & $83(62.9)$ & $2(28.6)$ & $9(69.2)$ & 0.167 \\
\hline Anastomotic leak & $11(8.3)$ & $1(14.3)$ & $1(7.7)$ & 0.646 \\
\hline Hospital stay (>POD\#10) & $61(46.2)$ & $5(71.4)$ & $7(53.8)$ & 0.411 \\
\hline Histology (NA in 1) & & & & 0.051 \\
\hline WD & $17(13.0)$ & $2(28.6)$ & $0(0)$ & \\
\hline MD & $108(82.4)$ & $5(71.4)$ & $10(76.9)$ & \\
\hline PD or mucinous & $6(4.6)$ & $0(0)$ & $3(23.1)$ & \\
\hline TRG (NA in 2) $M G \geq 3$ & $93(71.5)$ & $6(85.7)$ & 12 (92.3) & 0.257 \\
\hline ypT4 & $6(4.5)$ & $1(14.3)$ & $2(15.4)$ & 0.124 \\
\hline Tumor size >2 cm & $78(59.1)$ & $5(71.4)$ & $9(69.2)$ & 0.697 \\
\hline Retrieved nodes <12 & $40(30.3)$ & $4(57.1)$ & $5(38.5)$ & 0.274 \\
\hline CRM $\leq 1 \mathrm{~mm}$ (NA in 16) & $14(11.9)$ & $1(20.0)$ & $4(30.8)$ & 0.108 \\
\hline LVI (+) & $10(7.6)$ & $0(0)$ & $1(7.7)$ & $>0.999$ \\
\hline R1 resection & $4(3.0)$ & $0(0)$ & $2(15.4)$ & 0.108 \\
\hline aCT regimen & & & & 0.381 \\
\hline 5-FU-based & $124(93.9)$ & $6(85.7)$ & NA & \\
\hline FOLFOX & $7(5.3)$ & $1(14.3)$ & NA & \\
\hline Unknown & $1(0.8)$ & $0(0)$ & NA & \\
\hline
\end{tabular}

Values are presented as number (\%).

ASA PS, American Society of Anesthesiologists physical status; CEA, carcinoembryonic antigen; AV, anal verge; nCRT, neoadjuvant chemoradiotherapy; 5-FU, 5-fluorouracil; APR, abdominoperineal resection; POD, postoperative day; NA, nonapplicable; WD, well-differentiated; MD, moderately differentiated; PD, poorly differentiated; TRG, tumor regression grade; CRM, circumferential resection margin; LVI, lymphovascular invasion; aCT, adjuvant chemotherapy. 

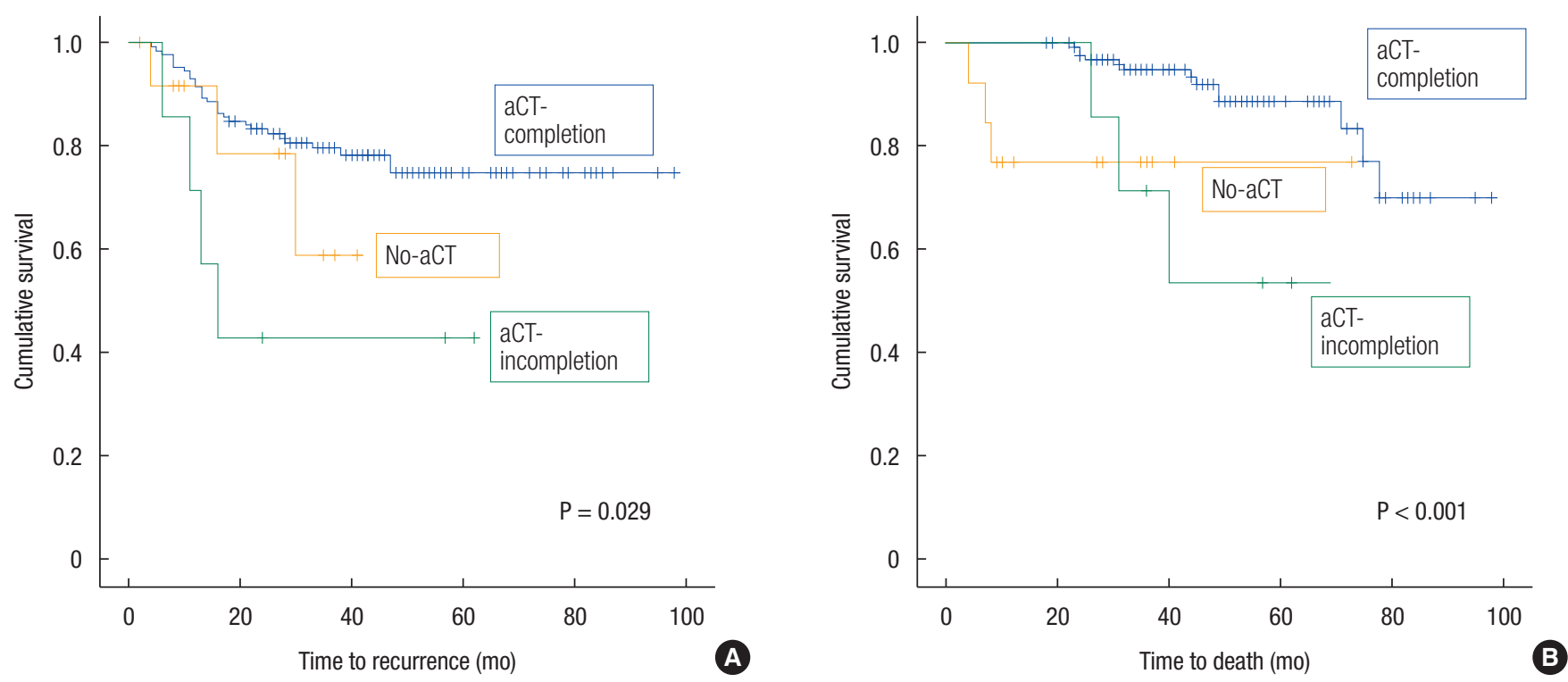

Fig. 1. (A) Survival curves of disease-free survival. (B) Survival curves of overall survival. aCT, adjuvant chemotherapy.

Table 2. Prognostic factors of disease-free survival

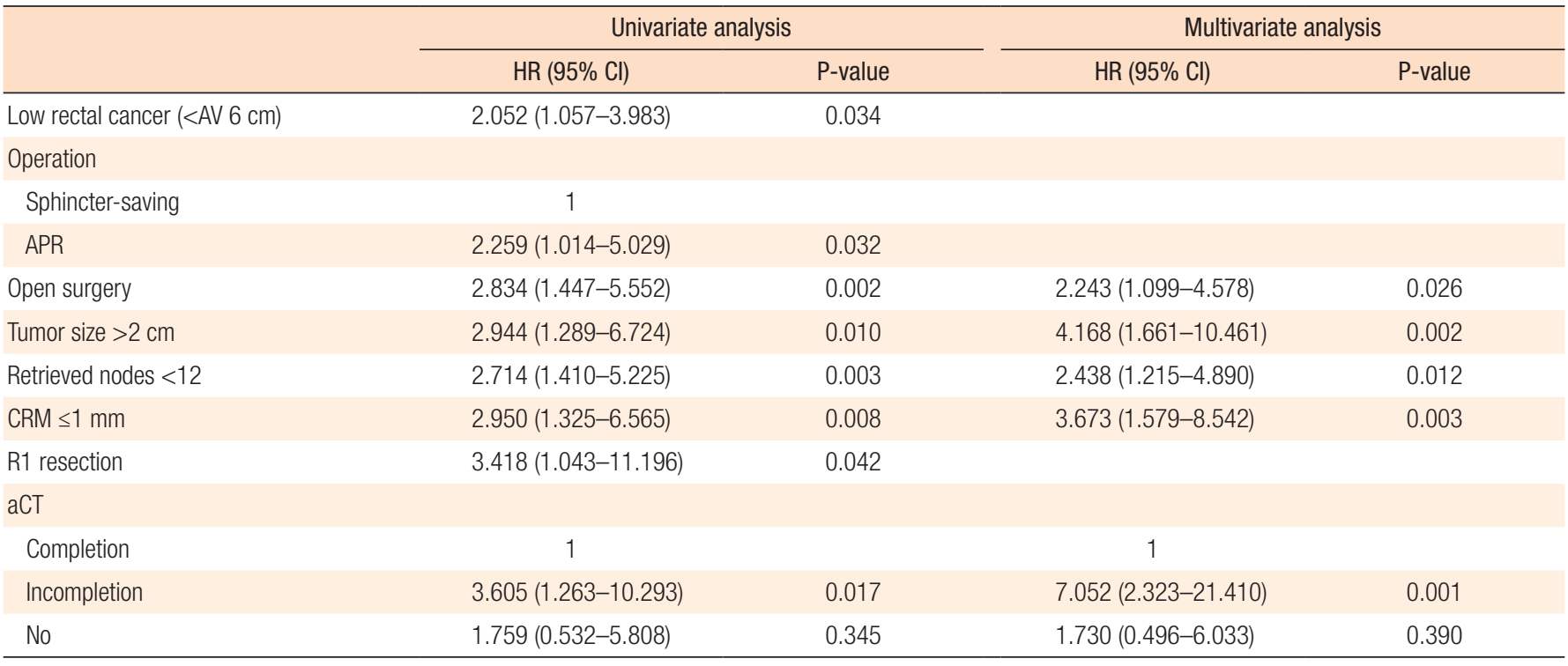

$\mathrm{HR}$, hazard ratio; $\mathrm{Cl}$, confidence interval; AV, anal verge; APR, abdominoperineal resection; CRM, circumferential resection margin; aCT, adjuvant chemotherapy.

surgery (HR, 20.889; 95\% CI, 3.684-118.455; P = 0.001), CRM positivity (HR, 3.965; 95\% CI, 1.313-11.976; $\mathrm{P}=0.015$ ), aCT incompletion compared to aCT completion (HR, 16.879; 95\% CI, 4.372-65.158; $\mathrm{P}<0.001)$ and no-aCT initiation compared to aCT completion (HR, 26.371; 95\% CI, 3.703-187.828; P = 0.001) were independent prognostic factors for poor OS (Table 3 ).

\section{DISCUSSION}

This study demonstrated that, compared with aCT completion,
aCT incompletion after nCRT and TME was associated with poor DFS and OS, and lack of aCT initiation was also associated with poor OS in ypstage 2 rectal cancer patients. To the best of our knowledge, this is the first study to investigate the association between completion of planned aCT and survival outcomes in rectal cancer patients treated with nCRT and radical surgery.

For locally advanced rectal cancer, nCRT is a standard treatment. Chemotherapeutic agents are used as radiosensitizers during nCRT, and the doses are not sufficient for systemic control. Moreover, the time interval between diagnosis and aCT initiation 
Table 3. Prognostic factors of overall survival

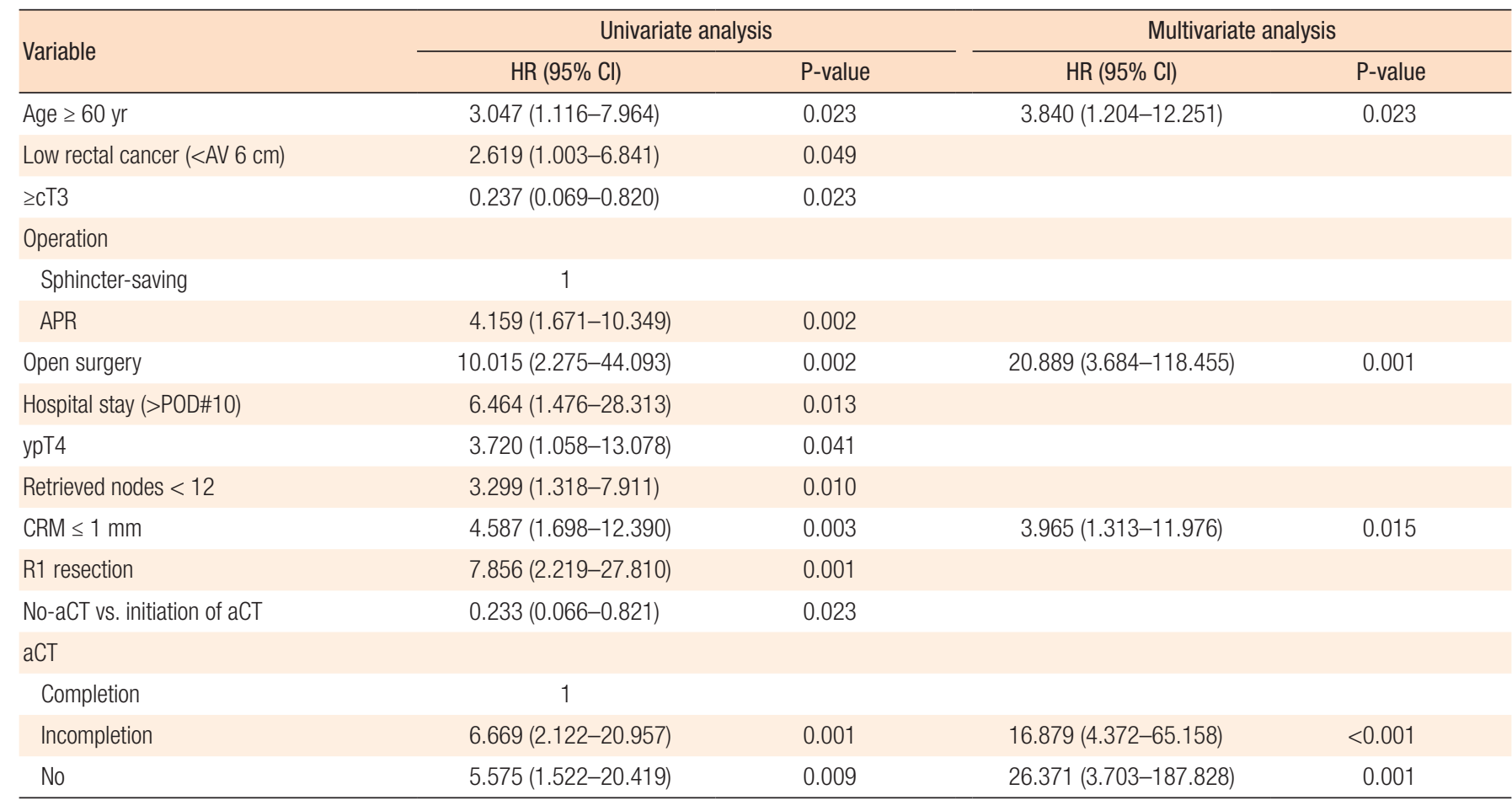

$\mathrm{HR}$, hazard ratio; $\mathrm{Cl}$, confidence interval; $\mathrm{AV}$, anal verge; APR, abdominoperineal resection; POD, postoperative day; CRM, circumferential resection margin; aCT, adjuvant chemotherapy.

is approximately $4-5$ months when long-course RT is applied. Therefore, a prolonged time interval between nCRT and surgery may result in failure to eradicate micrometastases by allowing subclinical tumor deposits to grow during the time required to achieve local control [8]. Many guidelines recommend perioperative treatment for 6 months in rectal cancer, so aCT is conventionally used for 4 months after nCRT and TME in pretreated rectal cancer patients. Given that the duration of planned aCT is shorter in rectal cancer than in colon cancer and that aCT initiation is delayed in pretreated rectal cancer patients, it is logical to question the oncologic impact of aCT incompletion, along with that of aCT initiation.

The use of aCT and failure to complete aCT have been associated with poor survival in stage 3 and high-risk stage 2 colon cancer patients in previous studies [18-20]. In contrast, the benefit and proper duration of aCT have not been determined in pretreated rectal cancer patients. Previous RCTs on aCT after neoadjuvant treatment in rectal cancer patients have reported no survival benefits [14-16]. The European Organization for Research and Treatment of Cancer 222921 trial initially indicated that aCT only benefited good responders exhibiting complete pathological remission or ypstage 1 cancer among pretreated rectal cancer patients; however, the 10-year follow-up results revealed no longterm survival benefits for these patients $[2,14]$. The long-term results from an Italian randomized trial (I-CNR-RT) and the Dutch
Colorectal Cancer Group (randomized phase III trial (PROCTOR-SCRIPT trial) also demonstrated no benefit of aCT in patients who had undergone nCRT $[15,16]$. However, these studies included good responders with ypN0 cancer, poor responders with ypN+ cancer and patients who did not complete their planned aCT schedules, exhibiting low compliance rates. Thus, the efficacy of aCT $(42.9 \%-73.6 \%)$ might have been underestimated [14-16].

Recent retrospective studies have attempted to identify specific subgroups who might benefit from aCT among rectal cancer patients who had undergone nCRT; however, these studies have had conflicting results $[10-13,17]$. The subjects were mostly good responders with ypN0 cancer [10-13]. However, the completion status of planned aCT was not clarified in most of these studies [10-12].

Few studies have focused on ypstage 2 patients. In such patients, the omission of aCT might raise concerns about a poor prognosis, because these patients initially had locally advanced disease, and still exhibited an advanced ypT stage at final pathology, despite displaying node negativity. In one retrospective study that attempted to identify subgroups that would benefit from aCT, no difference in DFS was found among ypstage 2 patients, despite benefits from aCT in the whole population (ypTanyNanyM0) [17].

When the no-aCT group was used as a reference, the aCT-in- 
completion group did not exhibit any difference in OS (HR, 0.640; 95\% CI, 0.086-4.760; P = 0.663); however, the aCT-completion group exhibited better OS (HR, 0.038; 95\% CI, 0.005-0.270; P = 0.001). In addition, when compared to the aCT-completion group, the aCT-incompletion group displayed worse DFS and OS, and the no-aCT group exhibited worse OS. Therefore, based on these findings, it is essential to increase compliance with aCT initiation and ensure the completion of all planned cycles by encouraging patients and properly managing aCT-induced toxicity.

This study had some limitations due to its retrospective nature, such as possible selection bias and type 2 error. The sample size included in the analyses was small, with relatively large data censorship. If more cases of aCT-incompletion and no-aCT had been included, the statistical significance of the demographics of the 3 groups may have been different, leading to a different interpretation of the results. Furthermore, the patients were heterogeneous with respect to their nCRT and aCT regimens; thus, our results cannot be completely applied in clinical practice. Therefore, it is imperative to conduct RCTs to collect firm evidence on the proper aCT duration that will improve the oncologic outcomes of pretreated rectal cancer patients.

In conclusion, this study demonstrated that only the aCT-completion group benefited from aCT after $\mathrm{nCRT}$ and TME; the no$\mathrm{aCT}$ and aCT-incompletion groups had poor prognoses.

In patients with ypstage 2 rectal cancer, aCT after nCRT and TME affected both DFS and OS; however, aCT incompletion did not have any survival benefit, implying that the initiation of aCT alone does not improve oncologic outcomes. Only aCT completion appears to have survival benefits in pretreated rectal cancer patients. Therefore, it is desirable to improve patient compliance with the completion of aCT, in light of its survival benefits in patients with ypstage 2 rectal cancer.

\section{CONFLICT OF INTEREST}

No potential conflict of interest relevant to this article was reported.

\section{ACKNOWLEDGMENTS}

This work was supported by an Inha University Research Grant (INHA-57831).

\section{REFERENCES}

1. Sauer R, Becker H, Hohenberger W, Rödel C, Wittekind C, Fietkau R, et al. Preoperative versus postoperative chemoradiotherapy for rectal cancer. N Engl J Med 2004;351:1731-40.

2. Bosset JF, Collette L, Calais G, Mineur L, Maingon P, RadosevicJelic L, et al. Chemotherapy with preoperative radiotherapy in rectal cancer. N Engl J Med 2006;355:1114-23.

3. Gérard JP, Conroy T, Bonnetain F, Bouché O, Chapet O, Closon-
Dejardin MT, et al. Preoperative radiotherapy with or without concurrent fluorouracil and leucovorin in T3-4 rectal cancers: results of FFCD 9203. J Clin Oncol 2006;24:4620-5.

4. Sebag-Montefiore D, Stephens RJ, Steele R, Monson J, Grieve R, Khanna $S$, et al. Preoperative radiotherapy versus selective postoperative chemoradiotherapy in patients with rectal cancer (MRC CR07 and NCIC-CTG C016): a multicentre, randomised trial. Lancet 2009;373:811-20.

5. Bujko K, Nowacki MP, Nasierowska-Guttmejer A, Michalski W, Bebenek M, Kryj M. Long-term results of a randomized trial comparing preoperative short-course radiotherapy with preoperative conventionally fractionated chemoradiation for rectal cancer. Br J Surg 2006;93:1215-23.

6. Valentini V, Lambin P, Myerson RJ. Is it time for tailored treatment of rectal cancer? From prescribing by consensus to prescribing by numbers. Radiother Oncol 2012;102:1-3.

7. Quasar Collaborative Group, Gray R, Barnwell J, McConkey C, Hills RK, Williams NS, et al. Adjuvant chemotherapy versus observation in patients with colorectal cancer: a randomised study. Lancet 2007;370:2020-9.

8. Glimelius B. On a prolonged interval between rectal cancer (chemo)radiotherapy and surgery. Ups J Med Sci 2017;122:1-10.

9. Poulsen LØ, Qvortrup C, Pfeiffer P, Yilmaz M, Falkmer U, Sorbye H. et al. Review on adjuvant chemotherapy for rectal cancer why do treatment guidelines differ so much? Acta Oncol 2015;54: 437-46.

10. Fietkau R, Barten M, Klautke G, Klar E, Ludwig K, Thomas H, et al. Postoperative chemotherapy may not be necessary for patients with ypN0-category after neoadjuvant chemoradiotherapy of rectal cancer. Dis Colon Rectum 2006;49:1284-92.

11. Kim CG, Ahn JB, Shin SJ, Beom SH, Heo SJ, Park HS, et al. Role of adjuvant chemotherapy in locally advanced rectal cancer with ypT0-3N0 after preoperative chemoradiation therapy and surgery. BMC Cancer 2017;17:615.

12. Park IJ, Kim DY, Kim HC, Kim NK, Kim HR, Kang SB, et al. Role of adjuvant chemotherapy in ypT0-2N0 patients treated with preoperative chemoradiation therapy and radical resection for rectal cancer. Int J Radiat Oncol Biol Phys 2015;92:540-7.

13. Kiran RP, Kirat HT, Burgess AN, Nisar PJ, Kalady MF, Lavery IC. Is adjuvant chemotherapy really needed after curative surgery for rectal cancer patients who are node-negative after neoadjuvant chemoradiotherapy? Ann Surg Oncol 2012;19:1206-12.

14. Bosset JF, Calais G, Mineur L, Maingon P, Stojanovic-Rundic S, Bensadoun RJ, et al. Fluorouracil-based adjuvant chemotherapy after preoperative chemoradiotherapy in rectal cancer: long-term results of the EORTC 22921 randomised study. Lancet Oncol 2014;15:184-90.

15. Sainato A, Cernusco Luna Nunzia V, Valentini V, De Paoli A, Maurizi ER, Lupattelli M, et al. No benefit of adjuvant fluorouracil leucovorin chemotherapy after neoadjuvant chemoradiotherapy in locally advanced cancer of the rectum (LARC): long term results of a randomized trial (I-CNR-RT). Radiother Oncol 2014; 
113:223-9.

16. Breugom AJ, van Gijn W, Muller EW, Berglund Å, van den Broek $\mathrm{CB}$, Fokstuen T, et al. Adjuvant chemotherapy for rectal cancer patients treated with preoperative (chemo)radiotherapy and total mesorectal excision: a Dutch Colorectal Cancer Group (DCCG) randomized phase III trial. Ann Oncol 2015;26:696-701.

17. Jung KU, Kim HC, Park JO, Park YS, Park HC, Choi DH, et al. Adjuvant chemotherapy after neoadjuvant chemoradiation and curative resection for rectal cancer: is it necessary for all patients? J Surg Oncol 2015;111:439-44.
18. Morris M, Platell C, Fritschi L, Iacopetta B. Failure to complete adjuvant chemotherapy is associated with adverse survival in stage III colon cancer patients. Br J Cancer 2007;96:701-7.

19. Neugut AI, Matasar M, Wang X, McBride R, Jacobson JS, Tsai WY, et al. Duration of adjuvant chemotherapy for colon cancer and survival among the elderly. J Clin Oncol 2006;24:2368-75.

20. Dobie SA, Baldwin LM, Dominitz JA, Matthews B, Billingsley K, Barlow W. Completion of therapy by Medicare patients with stage III colon cancer. J Natl Cancer Inst 2006;98:610-9. 